\title{
Structural analysis of the EGFR TK domain and potential implications for EGFR targeted therapy
}

\author{
WEIWEI NIE ${ }^{1}$, LIN TANG $^{1}$, HAIYANG ZHANG $^{3}$, JIAQING SHAO $^{2}$, YUCAI WANG $^{1}$, \\ LONGBANG CHEN $^{1}$, DONGHAI $\mathrm{LI}^{3}$ and XIAOXIANG GUAN ${ }^{1}$

\begin{abstract}
Departments of ${ }^{1}$ Medical Oncology and ${ }^{2}$ Endocrinology, Jinling Hospital, Medical School of Nanjing University, 305 East Zhongshan Road, Nanjing 210002; ${ }^{3}$ Jiangsu Engineering Research Center for MicroRNA Biology and Biotechnology, State Key Laboratory of Pharmaceutical Biotechnology, School of Life Sciences, Nanjing University, Nanjing 210093, P.R. China
\end{abstract}

Received November 16, 2011; Accepted January 9, 2012

DOI: $10.3892 /$ ijo.2012.1356

\begin{abstract}
The development and clinical application of TK domain inhibitors (TKIs) provide important insights into the broader field of cancer-targeted therapies. To discuss the recent advances in the atomic level understanding of EGFR TK domain mutations, we aim at highlighting the current and future importance of these studies on malignancies where the TK domain is improperly activated. The analysis is conducted on published TK domain crystal structures deposited in the Protein Data Bank, or homology structures generated by homology modeling and AutoDock 4.2 software using the program O. Mutations in exon 19 are the most common pathogenic mutations, so the crystal structures with these mutations are analyzed and compared in detail. In addition, we demonstrate how these crystal structures of EGFR conformation with TK domain mutations and those binding with small molecule inhibitors unveil the active or inactive mechanisms. As to the increasing resistance to the TKI, we summarize the progress on overcoming this challenge. Simultaneously, we predict the structure of BIKW-2992 binding to EGFR and compare it with the validated structure of HKI-272. It is hoped that a more accurate resistance mechanism would be found. In brief, we believe that this research will provide insights into EGFR targeted therapies.
\end{abstract}

Correspondence to: Dr Xiaoxiang Guan, Department of Medical Oncology, Jinling Hospital, Medical School of Nanjing University, 305 East Zhongshan Road, Nanjing 210002, P.R. China

E-mail: xguan@nju.edu.cn

Dr Donghai Li, Jiangsu Engineering Research Center for MicroRNA Biology and Biotechnology, State Key Laboratory of Pharmaceutical Biotechnology, School of Life Sciences, Nanjing University, Nanjing 210093, P.R. China

E-mail: donghaili@nju.edu.cn

Key words: EGFR, EGFR mutations, crystal structures, TKI

\section{Contents}

1. Introduction

2. The activation mechanisms of TK domain

3. The activating mechanism of somatic mutations

4. Primary resistance to EGFR TKI

5. Other resistance mechanisms to TKI

6. Conclusion

\section{Introduction}

The EGFR gene encompasses $118 \mathrm{~kb}$ of sequence on the short arm of human chromosome 7 and contains 28 exons, which encode 1186 amino acids (1). The EGFR protein can be divided into three domains: an extracellular ligand binding domain, a transmembrane domain and an intracellular domain. The intracellular domain contains a juxtamembrane (JM) segment, a kinase domain (TK) and a COOH-terminal tail (C-terminal tail) (2). As a member of tyrosine kinase receptor family, EGFRs form homo- or hetero-dimers with a variety of ligands, leading to phosphorylations of the intracellular tyrosine kinase domain. When activated, the kinases produce a cascade of responses to downstream signaling pathways including Ras/Raf/MAPK and PI3K/Akt/mTOR (3). Therefore, the EGFR plays a critical role in the regulation of cell growth, DNA synthesis and the expression of oncogenes. Therefore, in tumorigenesis of many human cancers individuals with EGFR gene mutations or aberrant relative signal pathway are found (4).

The most important region-TK domain can be divided into an N-terminal ATP-binding lobe (N-lobe) and a C-terminal substrate-binding lobe (C-lobe) (5). Whenever activated or mutated, the TK domain has striking changes in the conformation. The smaller N-lobe, largely constituted by $\beta$-sheet and the highly conserved $\alpha-\mathrm{C}$ helix, has a prominent role in conformational changes of the binding pocket. The $\mathrm{C}$-lobe is larger and mostly helical. ATP is proved to be bound the deep cleft between the lobes and located near a highly conserved N-lobe structure (the phosphate binding loop, or P-loop). The kinases exist in two extreme conformational states: on (active) or off (inactive) (6). It seems that the kinases are always in the extended conformation when activated. Activation loop, or A-loop, phosphorylated 
in the active state, provides a platform for the downstream proteins. P-loop contains a conserved glycine-rich/sequence motif (GXGX $\phi G)$ (7). Because the $\phi$ is usually tyrosine or phenylalanine, the glycine residues allow the P-loop to approach the phosphates of ATP very closely, as well as make the loop very flexible to bind with small molecule inhibitors via changing backbone interactions in the absence of ATP.

In general, the mutations of the EGFR gene focus on the first four exons (18 to 21) of the TK domain gene, which code the N-lobe and part of the C-lobe (8) (Fig. 1). EGFR mutations target the part close to the ATP-binding cleft including $\alpha-\mathrm{C}$ helix, the A-loop and the P-loop. The mutations can be categorized as: class (i) in-frame deletions in exon 19, class (ii) single-nucleotide substitutions that cause an amino alteration, and class (iii) in-frame duplications and/or insertions in exon 20. Class (i) mutations usually include amino acid residues Leu746 to Glu750 ( $\left.{ }^{\triangle} \mathrm{L} 746-\mathrm{G} 750\right)$ located at the N-terminus of the kinase domain C-helix. It accounts for approximately $44 \%$ of the active TK domains mutations. In class (ii) mutations the single point mutation in exon 21 that substitutes a leucine with an arginine at codon 858 (L858R) accounts for $41 \%$ of EGFR active mutations. Class (iii) mutations of in-frame duplications or insertions of exon 20 , are the remaining $5 \%$ of active mutations. There are also other active mutations with low frequency in tumors (9) (Fig. 1). Most active mutations are hypothesized to result in similar conformation changes. It is described that these mutations narrow the ATP-binding cleft so as to increase both gene activation and TKI sensitivity.

However, not all tumors accompanying activating mutations are associated with an enhanced response to TKI. Mostly the emergence of drug resistance is conferred by a second point mutation in the TK domain (10). To date, the secondary resistance mutations contain Thr790Met (T790M), Asp761Tyr (D761Y) (11), Ser 768 Ile (S768I) (12), Leu747Ser (L747S) (13) and Thr854Ala (T854A) (14). The T790M mutation is found in approximately $50 \%$ of all patients with acquired resistance to TKI (15), while other resistance mutations are relatively rare. Compared to the activating mutations, the resistance mutations are expected to have conformational changes to reduce both gene activation and TKI sensitivity. Preclinical studies are striving at this puzzle. However, the responses of many rare point mutations to TKI remain unknown.

There is a broad consensus on the mechanism of activating mutations that the stabilization of the hydrophobic regulatory spine promotes shift of the kinase towards the constitutively active kinase form, and thus have a dramatic effect on the regulation of the enzyme (16). Because different mutations lead to different sensitivities to TKI, we analyzed and compared all the available structures of EGFR, aiming at demonstrating how structural insights help our understanding of active and resistance mechanisms.

\section{The activation mechanisms of TK domain}

The activation of TK domain requires two procedures: stimulation of receptor catalytic activity and subsequent creation of docking sites for downstream signaling proteins (17). The homoor hetero-dimers of extracellular domain integrated with other ligands cause tyrosine domain autophosphorylation. There are two different types of EGFR targeted: monoclonal antibodies such as cetuximab and panitumumab (18), and tyrosine kinase inhibitors. Here we focus on the latter. We obtained 29 crystal structures for TK domain from the Protein Data Bank (PDB). All the available crystal structures of the TK domain have been published by seven groups in the PDB (19-24). To compare these structures in detail, we confirm the conclusion that the activity of protein kinases is regulated by the conformational state of the catalytic domain. In the active state, the catalytic domain conformation governs the ability to transfer a phosphate from ATP to peptide substrate, and thus controls the downstream signaling pathway (7). There are two essential factors for activation: making the correct amino acid residues oriented and keeping peptide substrate binding site available. In the active state, the A-loop is extended away from the cleft to allow peptide substrate binding, while a catalytic glutamate residue (part of the C-helix) forms an ionic interaction with a lysine residue that coordinates the $\alpha$ and $\beta$ phosphates of ATP. When inactive, the A-loop often changes conformation dramatically to preclude the binding of peptide substrate, while the $\mathrm{C}$-helix rotates away, pulling with the critical catalytic glutamate residue modified by phosphorylation and dephosphorylation events. It is also proposed that the A-loop folds into a helix similar to the inactive CDK and Src family crystal structures, which prevents C-helix rotation toward the catalytic cleft (18). This can be interpreted that they all belong to the protein kinase family.

The EGFR dimer involves the contacts not only between the two extracellular domains but also between the kinase domains. Recent studies suggest that there is a ligand-independent mechanism for TK domain activation (25). Intracellularly, the $\mathrm{N}$-terminal lobe interacts with the C-terminal lobe forming an asymmetric dimer for activation. Moreover, the C-terminal half of the juxtamembrane segment latches the activated kinase domain to the activator, and the $\mathrm{N}$-terminal half of this segment further potentiates dimerization, likely by forming an antiparallel helical dimer that engages the transmembrane helices of the activated receptor. The formation of the activating juxtamembrane latch is prevented by the $\mathrm{C}$-terminal tail inactive kinase domain dimer. The analysis of EGFR mutations in cancer patients has shown that deletion of the C-terminal tail can drive constitutive EGFR activation (26). In addition, the TK domain can be activated by interacting with small proteins, such as Gab1 (27). This proteinprotein interactions also produce resistance to target therapy.

\section{The activating mechanism of somatic mutations}

As mentioned above, the kinase activity of EGFR is mostly dependent on the conformation of the catalytic domain. When mutated, the shift of the helical axis narrows the ATP-binding cleft and increases both gene activation and TKI sensitivity (4). Thus, the activating mutations located in the catalytic domain cause notable alternations of the structure.

One of the most frequent mutations in exon 19 is deletion that removes residues 746-750 of the expressed protein (28). We obtained the structure of EGFR with ${ }^{\triangle}$ E746-A750 mutation in exon 19 by homology modeling based in the structure of the active EGFR (PDB accession code 2gs2) (Fig. 2). Yet, none of those studies would have predicted the structure. We find that the orientation of the $\mathrm{N}$-terminal lobe relative to the C-terminal lobe is not very different in the two models. This is due to a rearrangement of the N-lobe in the inactive 


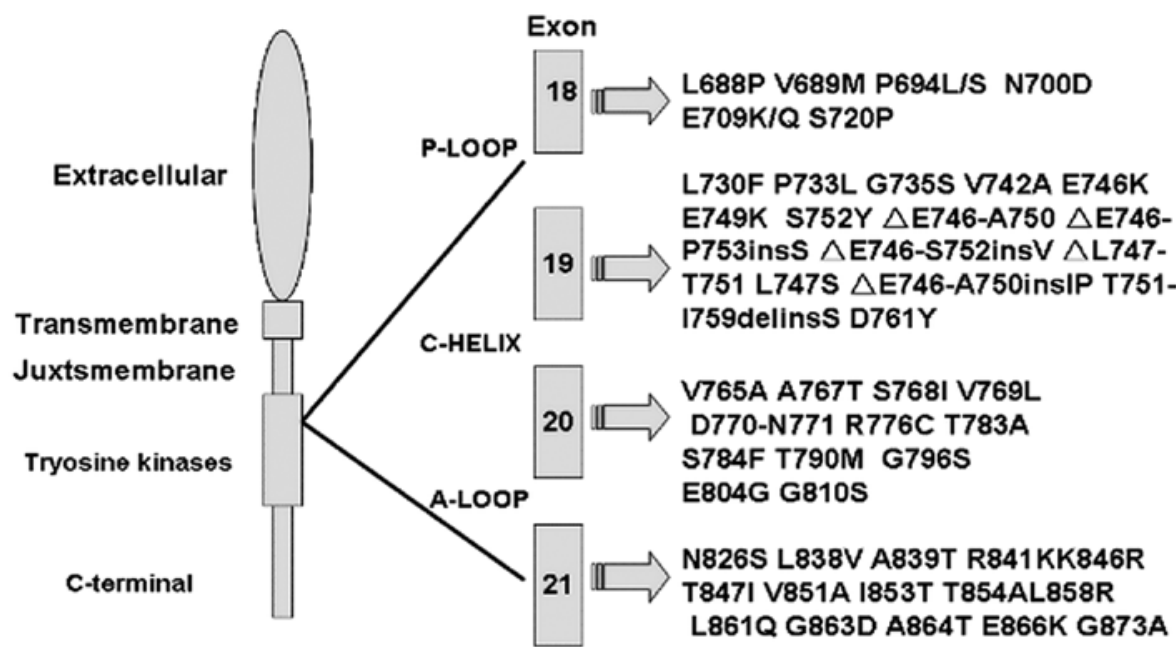

Figure 1. (Left) Schematic structure of EGFR, with the extra- and intracellular domains. (Right) Exon location and the vast majority of mutations in the tyrosine kinase mutations consist of missense mutations in exon 18, deletions in exon 19, duplications and/or insertions in exon 20 and a single-point mutation in exon 21. ${ }^{\triangle}$ L746-G750 mutation accounts for $44 \%$ of the active TK domains mutations. L858R mutation accounts for $41 \%$ of EGFR active mutations. The in-frame duplications or insertions of exon 20, are the remaining 5\% of active mutations. Other missense mutations: Asn826Ser (N826S), Leu838Val (L838V), Ala839Thr (A839T), Lys846Arg (K846R), Thr847Ise (T847I), Val851 (V851A), account for another 6\% of EGFR mutations.
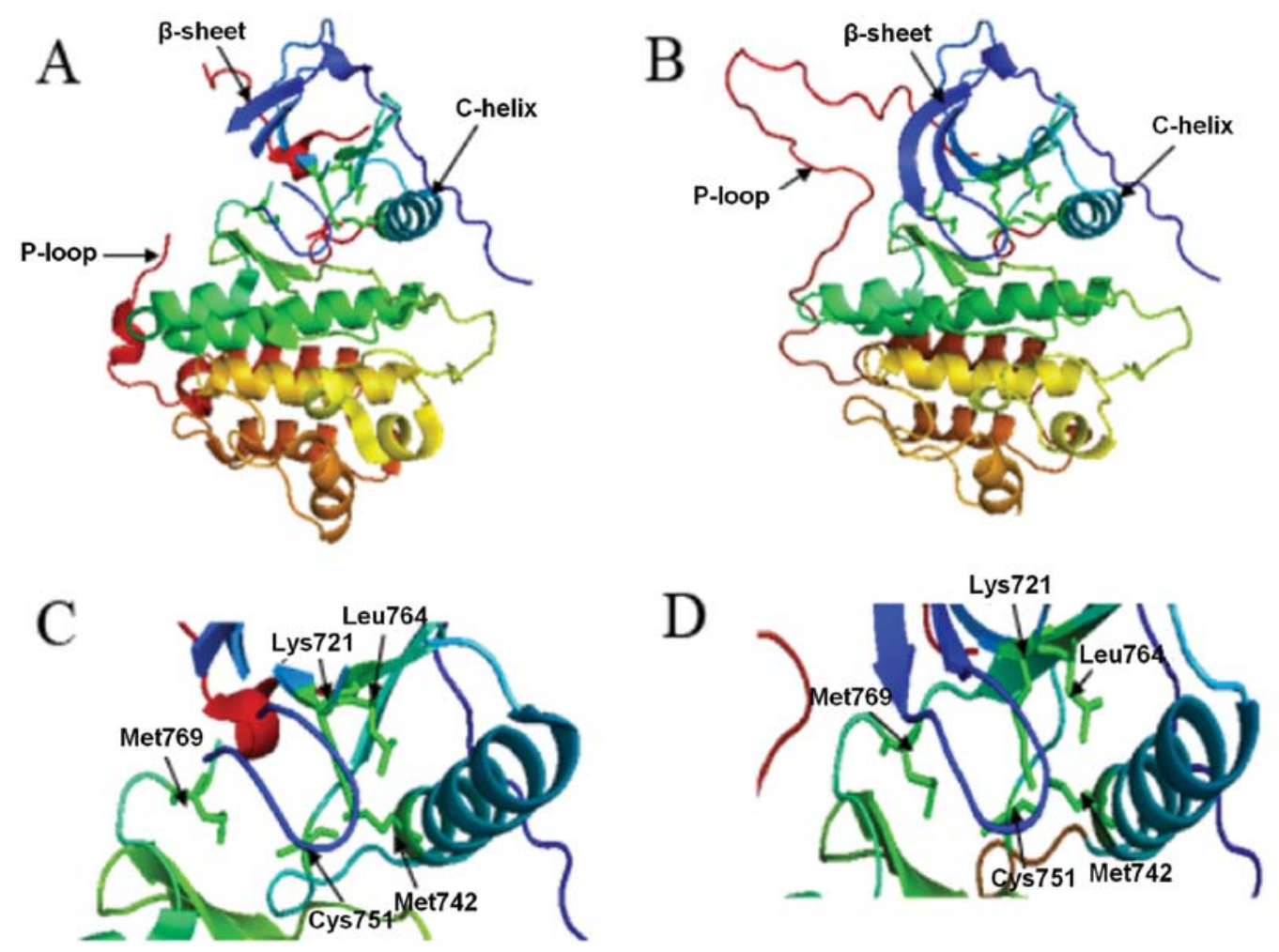

Figure 2. (A and B) Crystal structures of the active EGFR kinase domain (PDB:2gs2) and the active EGFR kinase domain with ${ }^{\triangle} \mathrm{E} 746$-A750 mutation, respectively. It shows that after mutation there is a change at the P-loop (shown in red) in the conformation. (C and D) A close-up view of the EGFR tyrosine kinase domain, with the critical amino acids in binding with ATP or the inhibitors. The pocket containing these critical amino acids has changed.

model during the molecular dynamics calculations. The major difference lies in the conformation of the long regulatory loop (residues 709-870) (29), which is highlighted in red. In the inactive state, this loop traverses the ATP binding site, which is located in the hydrophobic pocket including Lys 721, Met 742, Cys751, Leu764 and Met 769 (20). The loop is drawn taut, and sequentially the $\beta$-sheet and C-helix have changed, which leads to a more strained system (Fig. 2B). This change probably causes a loss of interactions between secondary structure subunits so as to destabilize the C-helix. Therefore, the deletion mutations in exon 19 increase the activation or sensitivity to various inhibitors. It demonstrates that the activity depends on the stability of the C-helix. Further functional experiments will offer a more complete understanding of this activating mutation.

The A-loop, typically with 20-30 residues in length, is located in exon 21 (30). Leu858 is within the helical turn, and 

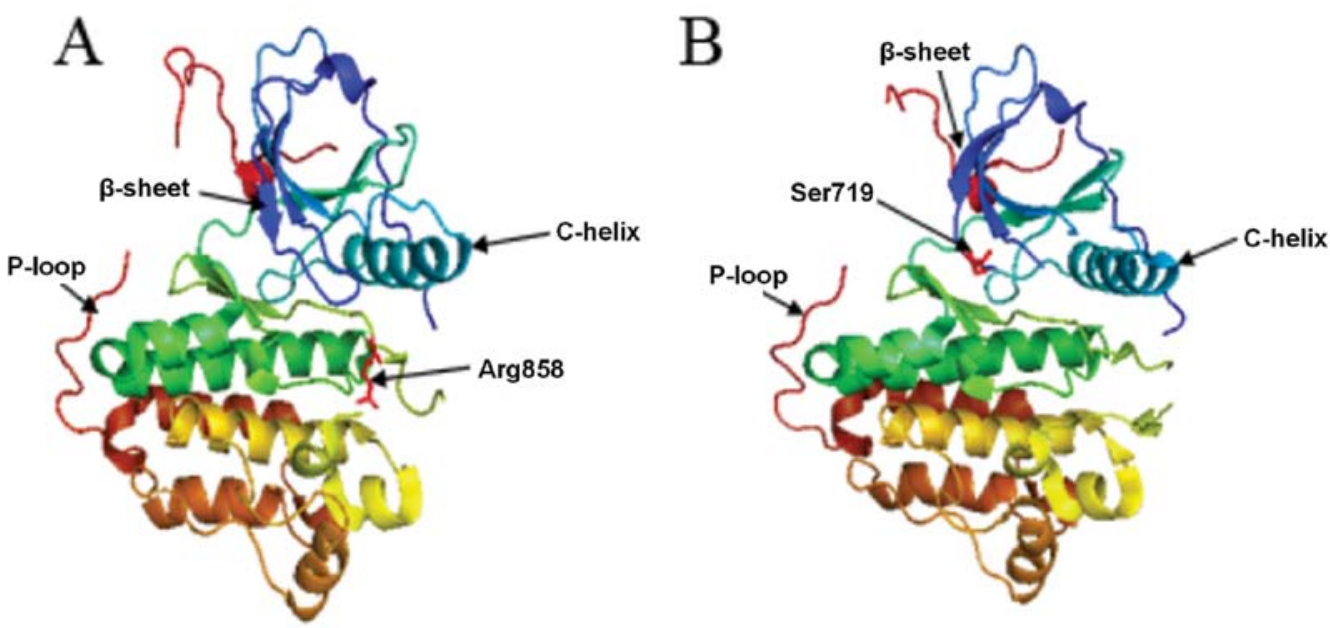

Figure 3. (A and B) Crystal structures of the active EGFR with L858R mutation (PDB:2itz) and the active EGFR kinase domain with G719S (PDB:2jit) mutation, respectively.

A

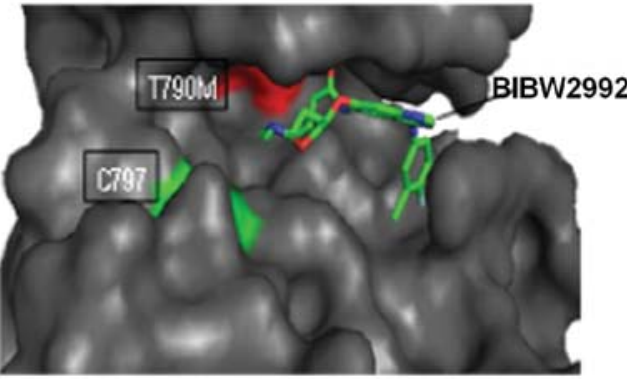

C

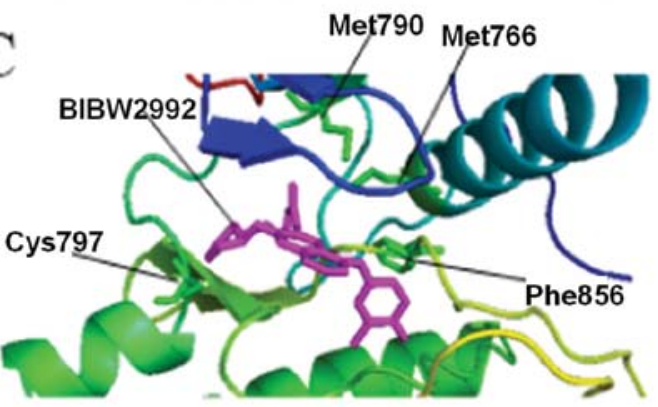

B
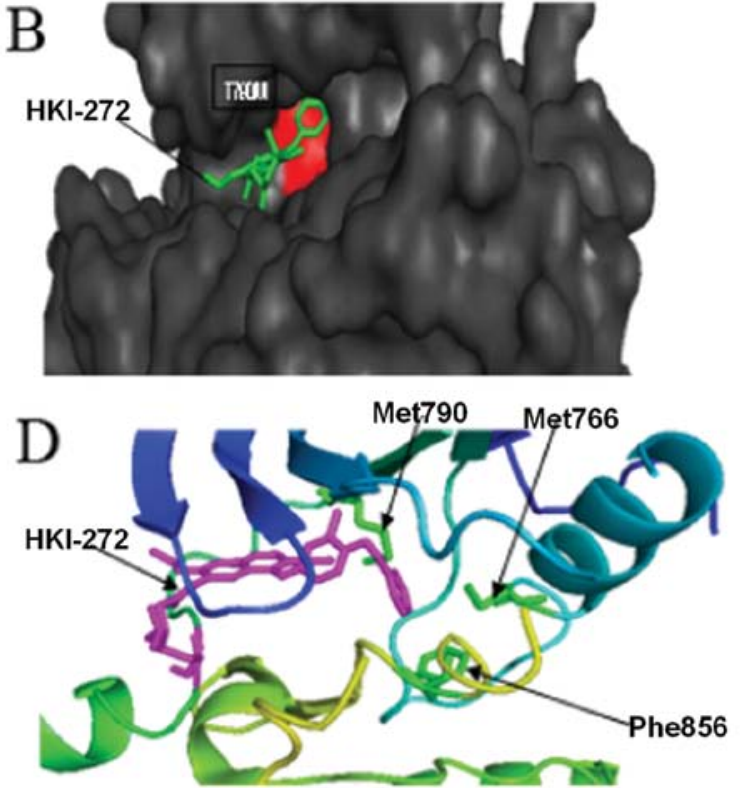

$\mathrm{E}$

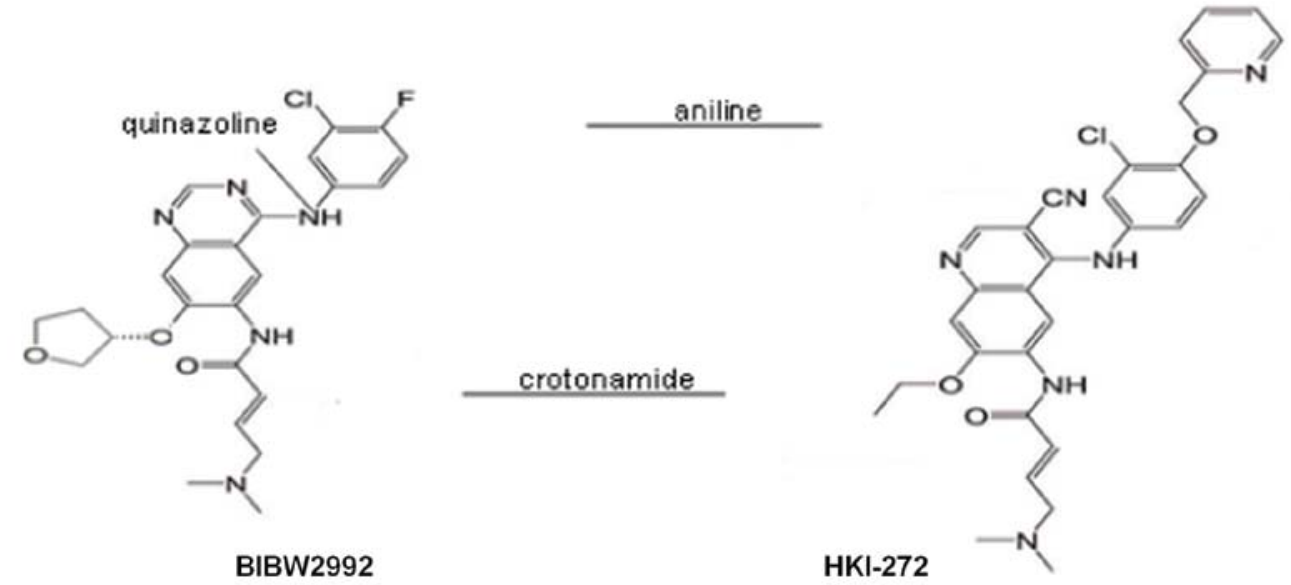

Figure 4. Structures of the T790M mutant in complex with HKI272 (PDB:2gs6) and BIBW2992 (B). With the same aniline structure, BIBW-2992 should bind to the inactive EGFR conformation as well. HKI-272 is surrounded by hydrophobic residues in the expanded pocket, including Met766 in the C-helix, Phe856 and Met790, while BIBW-2992 does not bind to Cys797 and is located in the pocket including Met766 in the C-helix, Phe856, and Met790 (C and D). In the two chemical structures of selected EGFR inhibitors, the compounds are drawn in a consistent orientation and conformation that reflects their approximate binding mode in the EGFR kinase (E). 
conducts key hydrophobic interactions with other residues in the N-lobe (Fig. 3). With the highest prevalence of single point activating mutations in the TK domain (31), L858R is located on the protein surface in the active state, while hides in a tightly packed hydrophobic pocket in the inactive (32). So it is expected that the replacement of a small hydrophobic leucine with a large polar argine will not only destabilize the inactive TK domain conformation but also stabilize the active conformation. Gly719 lies in the P-loop (Fig. 3). The conformation of the backbone of Gly719 is unique to glycine and inaccessible to other amino acids. The replacement of glycine with serine leads to this conformation significantly strained and sequentially makes P-loop adopt another conformation (33).

The changes of conformation are various in different mutations. The sensitivities of active mutations to small molecule inhibitors are not equal (34). Biochemical examinations indicated that cells harboring L858R, E746-A750 deletion, and G719S mutations are clearly more sensitive to gefitinib than wild-type cells (35). In clinical research, patients with deletion mutations gain a longer time to progression and median survival time than patients with L858R mutation $(\mathrm{P}=0.01)$. Deletion mutations predict better response to gefitinib than the L858R mutation (36). However, the L858R mutant cells are significantly more sensitive to gefitinib than the G719S mutants (32). Therefore, we conclude the deletion mutations in exon 19 have better sensitivity to EGFR-TK target therapy. A possible explanation is that the kinase activity is mostly dependent on the conformation of the catalytic domain. We deduced that the alteration of P-loop emerges as a prominent mechanism to regulate the kinase activity. The structures of EGFR with mutation in exon 19 are the most strained, which leads to the highest activity.

In the crystal structures of gefitinib bound to the L858R mutant, the rotation of gefitinib-alanine ring is by approximately 180 degrees (37). So it is possible that this alternate mode offers a tighter affinity of gefitinib for L858R mutant compared with wild-type EGFR. The structures of the L858R and G719S mutants with gefitinib also suggest that these mutations have improved $\mathrm{K}_{\mathrm{d}} / \mathrm{K}_{\mathrm{m}}$, ATP (34). Whether the same results can be found in exon 19 requires further study. Only by revealing the activating mechanism of the most common mutations, can we attain better therapy efficacy in the clinic.

\section{Primary resistance to EGFR TKI}

There are more and more mutations leading to increased resistance. Two strategies for preventing or overcoming this resistance have been found: to identify novel agents binding to and inhibiting TK domain by a distinct, non-ATP competitive mechanism, or to inhibit ATP binding to the TK domain irreversibly (38). The early studies expected that the irreversible binding compounds might show a longer duration of activation (37). A further motivation for designing these irreversible inhibitors is thought to realize the first path in the way of being noncompetitive with ATP. The resistance mechanism of T790M mainly is debated.

As the most common secondary mutation, T790M mutation seems to occur in approximately half of all patients with TKI resistance. Since Thr790 is involved in the binding site of TKI, the substitution of threonine to methionine causes steric hindrance of TKI binding (36), but T790M mutation increases the affinity to ATP as well (39), which leads to reduced potency of any ATP-competitive agent. The irreversible EGFR inhibitors, including BIBW-2992 (Afatinib) and HKI-272 (Neratinib), can inhibit growth in gefitinib-resistant NSCLC or Ba/F3 cell lines that contain the T790M mutation (40).

HKI-272 is a 4-(arylamino) quinoline-3-carbonitrile compound and a potent inhibitor of both EGFR and Her2 kinases (41). In complex with HKI-272, the EGFR kinase adopts an inactive conformation. In the inactive state, the regulatory $\mathrm{C}$-helix is displaced from its active position, which creates enlarged hydrophobic pocket to accommodate the bulky aniline substituent found in HKI-272. Like aniline quinazoline compounds, the quinoline core of HKI-272 forms a single hydrogen bond with the hinge region of the kinase. The 2-pyridinyl group of HKI-272 is surrounded by hydrophobic residues in the expanded pocket, including Met766 in the C-helix, Phe856 and Met790. The nitrile substituent of HKI-272 also approaches the above gatekeeper residue.

BIBW-2992 is also a dual inhibitor of both EGFR and ErbB2 kinases (41). It shows a higher affinity for binding to EGFR in the T790M resistance mutant (42). In a phase II clinical trial, BIBW-2992 attained responses in patients with deletion mutations in exon 19, L858R, L858R/T790M, L861Q, T854A (14) and G719S/S768I mutations.

It is reported that BIBW-2992 has increased efficacy over erlotinib, gefitinib and laptinib. Using AutoDock 4.2, we obtained the structure of T790M mutation in complex with BIBW-2992 (Fig. 4). BIBW-2992 does not bind to Cys797, but is located in the pocket including Met766 in the C-helix, Phe856, and Met790. The binding energy is $-4.36 \mathrm{kcal} / \mathrm{mol}$. HKI-272 is thought to bind to the inactive conformation of EGFR because of the additional aniline substitutions (13). With the same aniline structure, BIBW-2992 should bind to EGFR in the inactive conformation. Results from many studies have demonstrated that BIBW-2992 has a prolonged duration of activation in accordance with its mode of binding.

\section{Other resistance mechanisms to TKI}

In addition to the secondary mutations of EGFR, other resistance mechanisms such as KRAS mutations, C-met proto-oncogene (c-Met) amplification and mutations of downstream signal pathway molecules have been found.

KRAS mutations have been demonstrated to be associated with primary resistance to TKI in many tumors (38). KRAS mutations produce impaired GTPase activity and continual activation of Ras signaling, leading to the activation of proliferative and anti-apoptotic pathways (43). The most common mutations are located in exon 12 and 13. Activation of KRAS triggers both MAPK and MAPK-independent signal transduction (44). Oncogenic KRAS can promote tumor cells proliferation through Ras/Raf/MAPK pathway. Yet activating mutations of KRAS induce the constitutive MAPK-independent signal pathway and cause reduced sensitivity to EGFR target therapy.

c-Met amplification is detected in $20 \%$ of NSCLC patients with TKI resistance (half with T790M) (45). c-Met interacted with different signal modifiers, including scaffolding adaptors, cytoskeleton connectors and structurally homologous co-receptors leading to efficient activation of downstream signal 
transduction pathways that include MAPK, Jun amino-terminal kinases (JNK) and PI3K/Akt, signal transducer and activator of transcription proteins (STAT) (46). Thus, c-Met plays a critical role in cell proliferation, survival and migration. Because c-Met amplification is identified in a proportion of patients with acquired resistance to gefitinib/erlotinib, novel c-Met/TK inhibitors could be a treatment strategy for a small fraction of patients with c-Met amplification as the main mechanism of resistance (47). Trials with c-Met inhibitors (such as XL184, ARQ 197, PF-2341066, SGX523) will be discussed later (48).

Research over the past 40 years has uncovered crucial players in the EGFR signal transduction pathway, which can be roughly divided into two categories: the pro-survival arm of the pathway comprising the PI3K/Akt/mTOR cascade, and the proliferative arm consisting of the Ras/Raf/MAPK pathway (49). The importance of these signal pathways is well known. Deregulation of the PI3K/Akt pathway is associated with resistance to TKI in SCCHN (32). This offers another therapy target to overcome the resistance.

As a whole, it is a complex apparatus including KRAS mutations, c-Met amplification and the abnormal downstream signal pathways that produce other resistance to TKI. To take combination inhibitors to this complex network may overcome various resistances.

\section{Conclusion}

As a member of tyrosine kinase family, EGFR plays a critical role in the regulation of cell growth, DNA synthesis and the expression of oncogenes. We have found various abnormalities in EGFR during many pathological developments, particularly mutations of EGFR. Therefore, EGFRs have become a hot therapeutic target, which contains monoclonal antibodies and tyrosine kinase inhibitors. The emergence of the drug resistance impels further studies of genuine mechanism to this treatment. Research on the conformation of this therapeutic target will be of importance. By summarizing achievements of many scholars, we try to elaborate this puzzle.

The dominated activating mechanism is that alternations of structure of TK domain offer activity to EGFR. N-lobe, C-lobe, P-loop and A-loop, which include $\beta$-sheet and $\alpha$-C helix, have a prominent role in conformational changes of the binding pocket. Normally, the catalytic domain conformation governs the ability to transfer a phosphate from ATP to peptide substrate, and thus controls the downstream signal pathway. The ATP-binding catalytic cleft lies between the $\mathrm{N}$-terminal (N-lobe) and $\mathrm{C}$-terminal (C-lobe) lobes. Key structures that are required for function and activation surrounding the cleft, include the $\alpha-\mathrm{C}$ helix, the A-loop and the P-loop. A-loop provides a platform for the downstream proteins, and P-loop approaches the phosphates of ATP. The first four exons (18 to 21) of the TK domain gene code the $\mathrm{N}$-lobe and part of the $\mathrm{C}$-lobe. The mutations of the four exons consequently govern this activation. In the clinic, the deletion mutations in exon 19 and the single point mutation in exon 21 are the most common in the pathological changes. On the base of a validated crystal structure, scholars have put forward that the replacement of a small hydrophobic leucine with a large polar argine will not only destabilize the inactive TK domain conformation but stabilize the active conformation in the L858R mutation, which proves the changes of the conformation in this therapeutic target is important (6). There are no crystal structures of deletion mutations in exon 19. In order to understand this most important mutation, we predict the structure of ${ }^{\triangle}$ L746-G750 with homology modeling. The modeling shows significant changes of conformation. Thus, we infer that structural changes of EGFR TK domain are the leading role of this therapeutic target.

Much progress has been made in understanding the mechanism of TKI resistance, such as secondary mutations, gene amplification and mutations of the EGFR downstream signal pathway. We mainly debate irreversible EGFR inhibitors. We predicted the structure of T790M mutation in complex with BIBW-2992. It is expected that the structure would be beneficial to overcome the resistance.

The crystal structures have already led to much clearer understanding of EGFR activating and resistance mechanisms. Such a crystallographic study research should prove the development of more effective treatment strategies through the availability of better-targeted drugs or rational combination strategies with a higher response, a better therapeutic window and a long-time disease control. Further structural and functional studies are required to gain a more complete understanding of the EGFR TK domain. The studies how TK domain recognizes the ATP and different molecules at the molecular level are required to design inhibitors for therapeutic use.

\section{Acknowledgments}

This project was supported by grants from National Natural Science Foundation of China (81141094, 30870962, 31000323), Natural Science Foundation of Jiangsu Province (BK2011656), specialized Research Fund for Doctoral Program of Higher Education of China (20100091120023, 20100091120026) and Fundamental Research Funds for the Central Universities (1095020823).

\section{References}

1. Cai C, Peng Y, Buckley M, et al: Epidermal growth factor receptor activation in prostate cancer by three novel missense mutations. Oncogene 27: 3201-3210, 2008.

2. Voldborg BR, Damstrup L, Spang-Thomsen M and Poulsen HS: Epidermal growth factor receptor (EGFR) and EGFR mutations, function and possible role in clinical trials. Ann Oncol 8: 11971206, 1997.

3. Shigematsu H, Lin L, Takahashi T, et al: Clinical and biological features associated with epidermal growth factor receptor gene mutations in lung cancers. J Natl Cancer Inst 97: 339-346, 2005.

4. Gazdar AF, Shigematsu H, Herz J and Minna JD: Mutations and addiction to EGFR: the Achilles 'heal' of lung cancers? Trends Mol Med 10: 481-486, 2004.

5. Robinson DR, Wu YM and Lin SF: The protein tyrosine kinase family of the human genome. Oncogene 19: 5548-5557, 2000.

6. Kumar A, Petri ET, Halmos B and Boggon TJ: Structure and clinical relevance of the epidermal growth factor receptor in human cancer. J Clin Oncol 26: 1742-1751, 2008.

7. Huse M and Kuriyan J: The conformational plasticity of protein kinases. Cell 109: 275-282, 2002.

8. Shigematsu H and Gazdar AF: Somatic mutations of epidermal growth factor receptor signaling pathway in lung cancers. Int $\mathrm{J}$ Cancer 118: 257-262, 2006.

9. Sharma SV, Bell DW, Settleman J and Haber DA: Epidermal growth factor receptor mutations in lung cancer. Nat Rev Cancer 7: 169-181, 2007.

10. Gazdar AF: Active and resistance mutations of EGFR inonsmall-cell lung cancer: role in clinical response to EGFR tyrosine kinase inhibitors active EGFR mutations in NSCLC. Oncogene 28: S24-S31, 2009. 
11. Balak MN, Gong Y, Riely GJ, et al: Novel D761Y and common secondary T790M mutations in epidermal growth factor receptormutant lung adenocarcinomas with acquired resistance to kinase inhibitors. Clin Cancer Res 12: 6494-6501, 2006.

12. Chen YR, Fu YN, Lin CH, et al: Distinctive activation patterns in constitutively active and gefitinib-sensitive EGFR mutants. Oncogene 25: 1205-1215, 2006.

13. Costa DB, Halmos B, Kumar A, et al: BIM mediates EGFR tyrosine kinase inhibitor-induced apoptosis in lung cancers with oncogenic EGFR mutations. PLoS Med 4: 1669-1680, 2007.

14. Bean J, Riely GJ, Balak M, et al: Acquired resistance to epidermal growth factor receptor kinase inhibitors associated with a novel T854A mutation in a patient with EGFR-mutant lung adenocarcinoma. Clin Cancer Res 14: 7519-7525, 2008.

15. John T, Liu G and Tsao M: Overview of molecular testing in nonsmall-cell lung cancer: mutational analysis, gene copy number, protein expression and other biomarkers of EGFR for the prediction of response to tyrosine kinase inhibitors. Oncogene 28: S14-S23, 2009.

16. Garriz A, Qiu HF, Dey M, Seo EJ, Dever TE and Hinnebusch AG: A network of hydrophobic residues impeding helix alpha $\mathrm{c}$ rotation maintains latency of kinase Gen2, which phosphorylates the alpha subunit of translation initiation factor 2. Mol Cell Biol 29: 1592-1607, 2009.

17. Hubbard SR: Structural analysis of receptor tyrosine kinases. Prog Biophys Mol Biol 71: 343-358, 1999.

18. Johnston JB, Navaratnam S, Pitz MW, et al: Targeting the EGFR pathway for cancer therapy. Curr Med Chem 13: 3483-3492, 2006.

19. Stamos J, Sliwkowski MX and Eigenbrot C: Structure of the epidermal growth factor receptor kinase domain alone and in complex with a 4-anilinoquinazoline inhibitor. J Biol 277: 46265-46272, 2002

20. Wood ER, Truesdale AT, McDonald OB, et al: A unique structure for epidermal growth factor receptor bound to GW572016 (Lapatinib): relationships among protein conformation, inhibitor off-rate, and receptor activity in tumor cells. Cancer Res 64 6652-6659, 2004.

21. Zhang X, Gureasko J, Shen K, Cole PA and Kuriyan J: An allosteric mechanism for activation of the kinase domain of epidermal growth factor receptor. Cell 125: 1137-1149, 2006.

22. Yun CH, Boggon TJ, Li Y, et al: Structures of lung cancerderived EGFR mutants and inhibitor complexes: mechanism of activation and insights into differential inhibitor sensitivity. Cancer Cell 11: 217, 2007.

23. Blair JA, Rauh D, Kung C, et al: Structure-guided development of affinity probes for tyrosine kinases using chemical genetics. Nat Chem Biol 3: 229-238, 2007.

24. Zhou W, Eran D, Chen L, et al: Crystal structure of EGFR 696-1022 T790M mutant covalently binding to WZ4002. Nature 462 : 1070-1074, 2009.

25. Jura N, Endres NF, Engel K, et al: Mechanism for activation of the EGF receptor catalytic domain by the juxtamembrane segment Cell 137: 1293-1307, 2009.

26. Frederick L, Wang XY, Eley G and James CD: Diversity and frequency of epidermal growth factor receptor mutations in human glioblastomas. Cancer Res 60: 1383-1387, 2000.

27. Rodrigues GA, Falasca M, Zhang Z, Ong SH and Schlessinger J: A novel positive feedback loop mediated by the docking protein Gab1 and phosphatidylinositol 3-kinase in epidermal growth factor receptor signaling. Mol Cell Biol 20: 1448-1459, 2000.

28. Zhang Z, Stiegler AL, Boggon TJ, Kobayashi S and Halmos B EGFR-mutated lung cancer: a paradigm of molecular oncology. Oncotarget 1: 497-514, 2010.

29. Ishikawa N, Daigo Y, Takano A, et al: Increases of amphiregulin and transforming growth factor- $\alpha$ in serum as predictors of poor response to gefitinib among patients with advanced non-small cell lung cancers. Cancer Res 65: 9176, 2005.
30. Mirza A, Mustafa M, Talevich E and Kannan N: Co-conserved features associated with cis regulation of ErbB tyrosine kinases. PLoS One 5: e14310, 2010.

31. Dixit A, Torkamani A, Schork NJ and Verkhivker G: Computational modeling of structurally conserved cancer mutations in the RET and MET kinases: the impact on protein structure, dynamics, and stability. Biophys J 96: 858-874, 2009.

32. Bikker JA, Brooijmans N, Wissner A and Mansour TS: Kinase domain mutations in cancer: implications for small molecule drug design strategies. J Med Chem 52: 1493-1509, 2009.

33. Rosell R, Taron M, Reguart N, et al: Epidermal growth factor receptor activation: how exon 19 and 21 mutations changed our understanding of the pathway. Clin Cancer Res 12: 7222-7231, 2006.

34. Riely GJ, Politi KA, Miller VA, et al: Update on EGFR mutations in non-small cell lung cancer. Clin Cancer Res 12: 7232-7241, 2006.

35. Mitsudomi T and Yatabe Y: Mutations of the epidermal growth factor receptor gene and related genes as determinants of epidermal growth factor receptor tyrosine kinase inhibitors sensitivity in lung cancer. Cancer Sci 98: 1817-1824, 2007.

36. Jiang J, Greulich H, Janne PA, et al: Epidermal growth factorindependent transformation of $\mathrm{Ba} / \mathrm{F} 3$ cells with cancer-derived epidermal growth factor receptor mutants induces gefitinibsensitive cell cycle progression. Cancer Res 65: 8968-8974, 2005.

37. Yun CH, Mengwasser KE, Toms AV, et al: The T790M mutation in EGFR kinase causes drug resistance by increasing the affinity for ATP. Proc Natl Acad Sci USA 105: 2070-2075, 2008.

38. Nguyen KS, Kobayashi S and Costa DB: Acquired resistance to epidermal growth factor receptor tyrosine kinase inhibitors in nonsmall-cell lung cancers dependent on the epidermal growth factor receptor pathway. Clin Lung Cancer 10: 281-289, 2009.

39. Kwak EL, Sordella R, Bell DW, et al: Irreversible inhibitors of the EGFR receptor may circumvent acquired resistance to gefitinib. Proc Natl Acad Sci USA 102: 7665-7670, 2005.

40. Tsou HR, Overbeek-Klumpers EG, Hallett WA, et al: Optimization of 6,7-disubstituted-4-(arylamino) quinoline3 -carbonitriles as orally active, irreversible inhibitors of human epidermal growth factor receptor-2 kinase activity. J Med Chem 48: 1107-1131, 2005

41. Li D, Ambrogio L, Shimamura T, et al: BIBW2992, an irreversible EGFR/HER2 inhibitor highly effective in preclinical lung cancer models. Oncogene 27: 4702-4711, 2008.

42. Yang C, Shih J, Chao T, et al: Use of BIBW 2992, a novel irreversible EGFR/HER2 TKI, to induce regression in patients with adenocarcinoma of the lung and active EGFR mutations: preliminary results of a single-arm phase II clinical trial. J Clin Oncol 26: S430, 2008.

43. Riely GJ, Marks J and Pao W: KRAS mutations in non-small cell lung cancer. Proc Am Thorac Soc 6: 201-205, 2009.

44. Wicki A, Herrmann R and Christofori G: KRAS in metastatic colorectal cancer. Swiss Med Wkly 140: w13112, 2010.

45. Trusolino L, Bertotti A and Comoglio PM: Met signalling: principles and functions in development, organ regeneration and cancer. Nat Rev Mol Cell Biol 11: 834-848, 2010.

46. Bogoyevitch MA and Kobe B: Uses for JNK: the many and varied substrates of the c-Jun N-terminal kinases. Microbiol Mol Biol Rev 70: 1061-1095, 2006.

47. Porter J: Small molecule c-Met kinase inhibitors: a review of recent patents. Expert Opin Ther Pat 20: 159-177, 2010.

48. Tang Z, Du R, Jiang S, et al: Dual MET-EGFR combinatorial inhibition against T790M-EGFR-mediated erlotinib-resistant lung cancer. Br J Cancer 99: 911-922, 2008.

49. Ratushny V, Astsaturov I, Burtness BA, Golemis EA and Silverman JS: Targeting EGFR resistance networks in head and neck cancer. Cell Signal 21: 1255-1268, 2009. 